\title{
Free flowing and cohesive powders agitation in a cylindrical convective blender-kinetics experiments and Markov chain modelling
}

\author{
Léonard Legoix ${ }^{1}$, Mathieu Milhé ${ }^{1, *}$, Cendrine Gatumel $^{1}$, and Henri Berthiaux ${ }^{1}$ \\ ${ }^{1}$ Université de Toulouse, Centre RAPSODEE, CNRS UMR 5302, Ecole des Mines d'Albi, Campus Jarlard, 81013 Albi Cedex 09 , \\ France
}

\begin{abstract}
An original methodology for studying powder flow in a cylindrical convective blender has been developed. A free-flowing and a cohesive powder were studied, at a fixed stirring speed, in rolling regime. For both powders, three apparent flow mechanisms were evidenced: convection in the volume swept by the blades, diffusion/shearing between the agitated zone and the stagnant one, as well as in the stagnant zone itself, and avalanches at the powder bed surface between agitated and stagnant zones. After defining six zones in the blender, tracing experiments were carried out by placing appropriate tracers in different starting zones and sampling the whole bed at different stirring times, which lead to mixing kinetics of the powders into themselves. A Markov chains model of the blender allowed the quantification of the three mechanisms respective magnitude by fitting the experimental data. This simple model has a good agreement with the free-flowing powder data, but is not able to represent well the observations for the cohesive powder. Bed consolidation should probably be taken into account for this kind of powders and thus a linear Markov model is not sufficient.
\end{abstract}

\section{Introduction}

In spite of the recurring use of convective blenders in the pharmaceutical industry, their global modelling has not been carried out yet, particularly when focusing on fine or cohesive powders behaviour. A DEM approach is difficult to take on with particle sizes about $10 \mu \mathrm{m}$ leading to overall populations of $10^{14}$ when batches of $100 \mathrm{~kg}$ are considered.

Moreover, when dealing with batch planetary blenders, which combine two circular movements (namely rotation of the blades and gyration of their axis), the task is yet more cumbersome. For these reasons, a systemic approach has been undertaken in previous works, based on rheological observations at pilot scale [1]. This lead to the setup of a cylindrical convective blender (Fig. 1): this device allows carrying out rheological studies, flow observations and determination of mixing kinetics.

It should be noted that the term "mixing" is used in the sense of self-mixing of the powder in this study: the tracer employed for the mixing experiments are supposed to have the same flow behaviour and particle size than the powders studied.

The objective of this work is to study the differences in flow behaviour between a free-flowing and a cohesive powder when agitated by straight blades in a cylindrical convective blender, both experimentally and numerically thanks to a systemic modelling based on Markov chains.

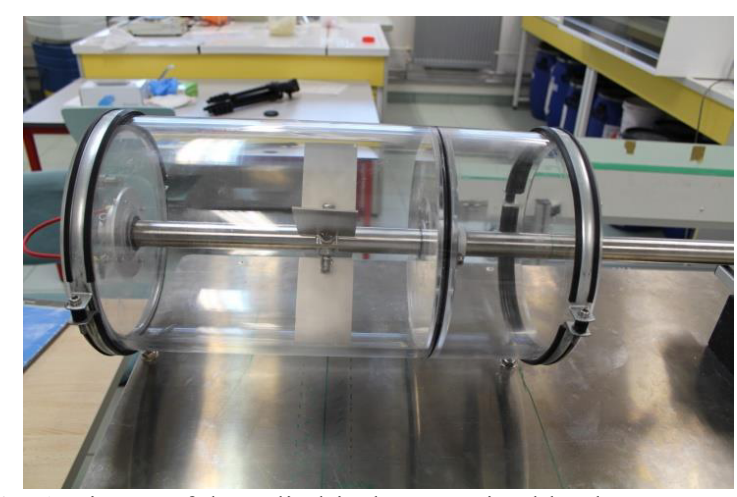

Fig. 1. Picture of the cylindrical convective blender

\section{Experimental determination of mixing kinetics}

\subsection{Powders studied}

The free-flowing powder used was semolina, the cohesive powder was lactose. Both have a polygonal shape, their physical characteristics are summed up in Table 1 . The mean diameter $d_{50}$ was determined by laser diffraction (Mastersizer3000, Malvern) under an air pressure of 3.5 bar, the Carr Index CI thanks to a volumenometer Erweka, according to eq. 1, where $\rho_{b}$ and $\rho_{t}$ are respectively the bulk density and the tapped density.

\footnotetext{
Corresponding author: mmilhe@mines-albi.fr
} 


$$
\mathrm{CI}=\left(\rho_{t}-\rho_{b}\right) / \rho_{t}
$$

Table 1. Powders characteristics

\begin{tabular}{|c|c|c|}
\hline Powder & Semolina & Lactose \\
\hline$d_{50}(\mu \mathrm{m})$ & 312 & 26 \\
\hline$\rho_{b}\left(\mathrm{~kg} / \mathrm{m}^{3}\right)$ & 679 & 495 \\
\hline$\rho_{t}\left(\mathrm{~kg} / \mathrm{m}^{3}\right)$ & 720 & 638 \\
\hline $\mathrm{CI}(\%)$ & 5.8 & 22.5 \\
\hline
\end{tabular}

\subsection{Experimental protocol}

Each experiment was performed with a stirring speed of $6 \mathrm{rad} / \mathrm{s}$, corresponding to a rolling regime (the powder flows along the rotating blades and is not projected). For both powders, the blender was filled at $83 \%$.

The blender sides were set asymmetrically and the volume was virtually divided into six zones of identical volume as illustrated in Fig. 2. Before an experiment, the blender was filled with the powder studied in five zones and with the corresponding tracer in the sixth zone.

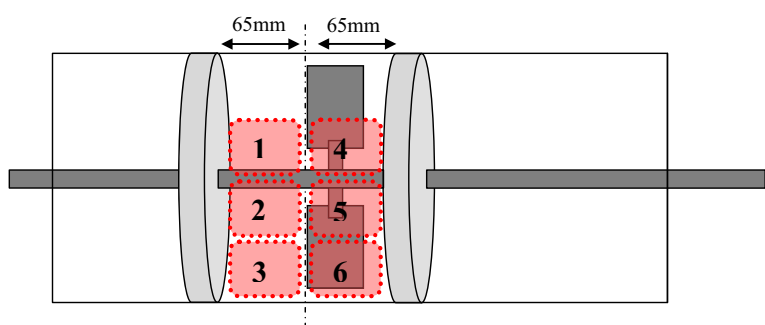

Fig. 2. Illustration of the 6 transversal zones considered in the blender

For each tracer initial position (zone 1, 3 and 4 in this study), three to four experiments were performed with different agitation durations. At the end of each experiment, the whole bed is sampled zone by zone, and the tracer mass fraction in each zone is determined (see next section).

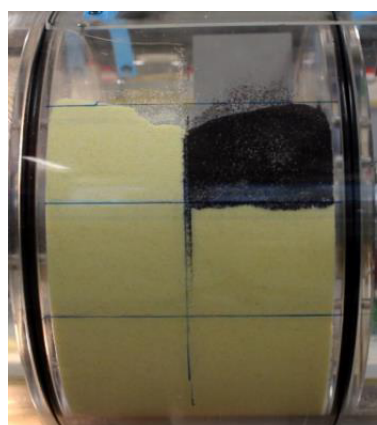

Fig. 3. Front view of the blender at the start of an experiment with the semolina tracer initially in zone 4.

Each series of experiment thus constitutes a mixing kinetics of the powder into itself, assuming the tracer behaves the same as the powder.

\subsection{Tracer analysis}

The tracer used for free-flowing experiments was semolina coloured thanks to an iodine ethanol solution: this tracer has the same $d_{50}$ than the original semolina and was detected by image analysis. A given zone sampled from the blender was homogenised and poured on a $19 \mathrm{~cm}$ diameter plate. 8 pictures were then taken at random locations with an x50 magnifying microscope and the images were analysed thanks to a Matlab $^{(}$ routine. The tracer concentration was obtained thanks to a calibration curve.

The tracer used for cohesive experiments was talc, since no colorimetric techniques proved satisfying for tracing purposes. The talc used had characteristics close to that of lactose. Its mass fraction in the samples was determined by thermogravimetry analysis: each sampled zone was homogenised and 12 samples of $2 \mathrm{~g}$ each were sampled randomly from it, before being heated at $650^{\circ} \mathrm{C}$ for $2 \mathrm{~h}$. The remaining talc was then weighed again and its initial mass fraction deduced since this compound showed no weight loss at this temperature.

\section{Markov chain modelling}

Powder mixing operations have been studied and modelled with Markov chains in previous works for continuous mixers [2,3] and batch mixers [4]. In this work, it was chosen to model the evolution of the tracer mass fraction in the six zones by a homogeneous and linear Markov chain: the transition probability from a zone (a state in Markov formalism) to another does not depend on the time or on the amount of tracer in this zone.

\subsection{Mechanisms considered}

Based on observations, four mass transfer mechanisms have been taken into account in this model:

- convection between zones 1 and 2, and between zones 2 and 3 , due to blade rotation.

- avalanching between zone 1 and 4, due to powder bed level differences after each blade pass.

- horizontal diffusion between zones 2 and 5, and between zones 3 and 6 .

- vertical diffusion between zones 1 and 2, and between zones 2 and 3 .

These mechanisms are respectively represented by the coefficients $c, a, d_{h}$ and $d_{v}$ hereafter. They represent the fraction of powder moving from a zone to another one due to the different mechanisms.

\subsection{Markov chain}

Let denote $S_{n}$ the vector describing the mass fraction of a tracer (or any extensive quantity related to the powder) in the different zones of the powder bed in the blender at the $n^{\text {th }}$ transition. According to the Markov property, the evolution of $S_{n}$ can be calculated from $S_{n-1}$ with eq. 2, where $\mathrm{P}$ is the matrix of transition probabilities. 


$$
S_{n}=P . S_{n-1}
$$

The matrix $P$ is constructed with the coefficients defined above (section 3.1): each term $P_{i, j}$ is the proportion of $S_{n}(j)$ moving to zone $i$ : it follows that it must be comprised between 0 and 1 . The diagonal terms $P_{j, j}$ are deduced from the other thanks to a mass balance, i.e. the sum of each column must be equal to 1 . The matrix $P$ is symmetric since all mechanisms are supposed to occur in both directions with the same magnitude.

$$
P=\left(\begin{array}{cccccc}
1-d_{v}-a & d_{v} & 0 & a & 0 & 0 \\
d_{v} & 1-2 d_{v}-d_{h} & d_{v} & 0 & d_{h} & 0 \\
0 & d_{v} & 1-d_{v}-d_{h} & 0 & 0 & d_{h} \\
a & 0 & 0 & 1-a-c & c & 0 \\
0 & d_{h} & 0 & c & 1-d_{h}-2 c & c \\
0 & 0 & d_{h} & 0 & c & 1-d_{h}-c
\end{array}\right)
$$

The characteristic time defining a transition is the time needed by the blades to make $1 / 4$ of a rotation: every $1 / 4$ of a rotation, the quantity described in $S_{n}$ is redistributed according to eq. 2 .

\subsection{Coefficients identification}

The four coefficients have been identified by fitting the model to the experimental mixing kinetics for both powders. Knowing the experimental mixing times $n_{\text {exp }}$ and the corresponding tracer mass fractions $S_{n}{ }^{\exp }$ for each experiment of a series with initial tracer position in zone $i$, the best coefficients are obtained by minimizing the objective function in eq. 4 .

$$
\Delta=\sum_{i=1,3,4}\left(\sum_{n_{\exp }}\left(S_{n}-S_{n}^{\text {exp }}\right)^{2}\right)
$$

\section{Results}

The experimental results are first used to obtain model coefficients for the two powders studied. For semolina, four experimental values of $S^{\text {exp }}$ were obtained, with agitation durations ranging from 4 to $300 \mathrm{~s}$. For lactose, only three values were obtained in the same range.

\subsection{Model coefficients}

The two sets of coefficients obtained for free-flowing semolina and cohesive lactose are gathered in Table 2. The coefficients $c$ and $d_{v}$ must be lower than 0.5 in order to avoid negative terms in $P$, which would be physically impossible. Both sets of coefficients show the same trend: convection is responsible for most part of mass transfer between the zones, while avalanches only concern about $3 \%$ of the powder in zones 1 and 4 . These two coefficients reflect the difference between the powders: the lactose is clearly less prone to flow due to its higher cohesion. This confirms the physical basis for this model, in spite of strong assumptions.

Diffusion mechanisms showed to have an influence 3 to 4 order of magnitude lower than convection by the blades. However, for semolina, the fact that $d_{h}$ is null is linked to the value of $c$ because $P_{5,5}=1-d_{h}-2 c$ and not necessarily because this particular mixing mechanism is not involved. The value of $d_{h}$ obtained for lactose is actually the accuracy of the optimization procedure used so it might not be reliable.

Table 2. Model coefficients for both powders

\begin{tabular}{|c|c|c|}
\hline Powder & Semolina & Lactose \\
\hline$c$ & 0.4999 & 0.4511 \\
\hline$a$ & 0.0347 & 0.0279 \\
\hline$d_{h}$ & 0 & 0.0001 \\
\hline$d_{v}$ & 0.0071 & 0 \\
\hline
\end{tabular}

\subsection{Free-flowing case}

Simulations and experimental data for semolina are compared in figures 4 and 5, corresponding respectively to initial tracer position in zones 4 and 1. Transition number is represented in logarithmic scale for visualization purposes. In both cases, the agreement is satisfying.
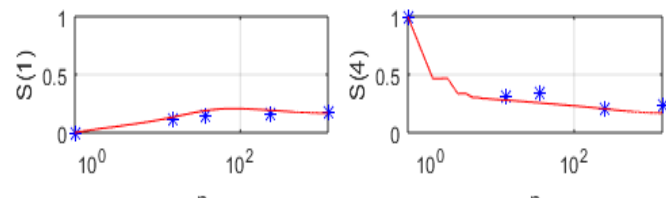

n
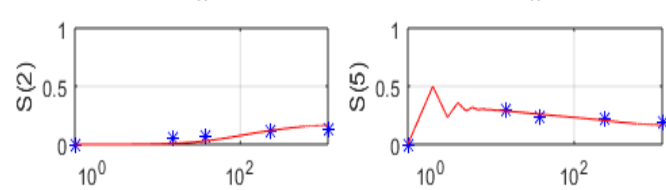

n
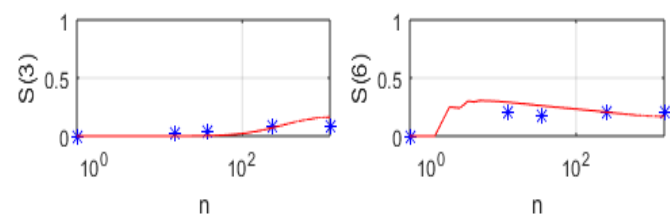

Fig. 4. Comparison of simulations and experimental data for semolina, with initial tracer position in zone 4

An oscillating behaviour can be observed in zones 4 to 6 during the first transitions, mainly because most of the powder in zone 5 is supposed to be exchanged with zones 4 and 6 at each transition. 

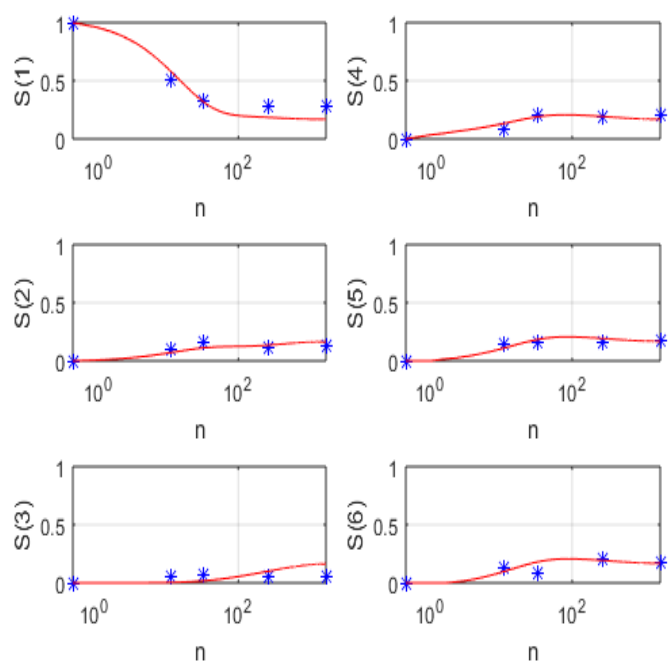

Fig. 5. Comparison of simulations and experimental data for semolina, with initial tracer position in zone 1

\subsection{Cohesive case}

Simulations and experimental data for lactose are compared in figures 6 and 7, with the same initial tracer positions. In this case, the agreement is less good, particularly when the tracer is initially in zone 1 . The set of coefficients identified does not allow describing correctly powder flows in the blender
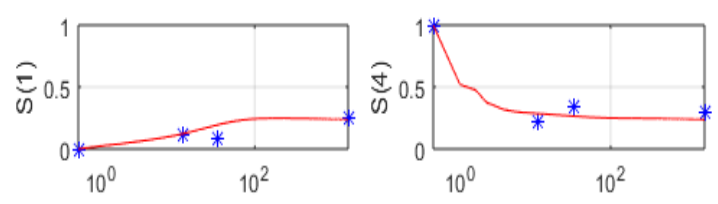

n

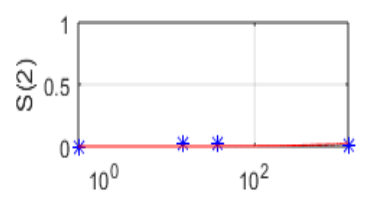

$\mathrm{n}$
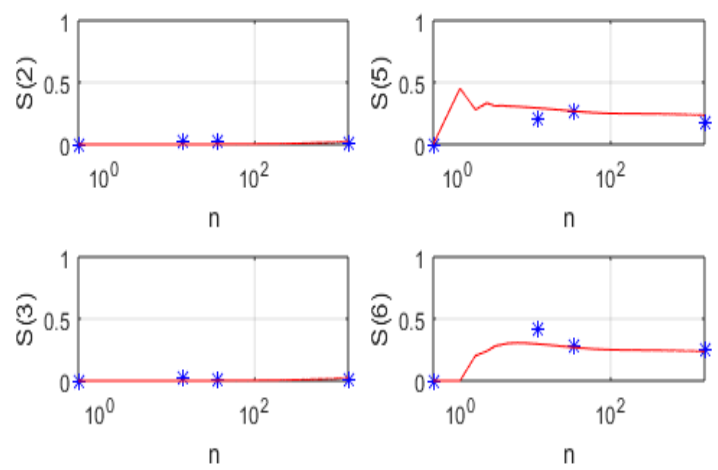

Fig. 6. Comparison of simulations and experimental data for lactose, with initial tracer position in zone 4

While experimental data show a stationary behaviour after the very first transitions, the model suggests a longer evolution and a better homogenization of the tracer between zones 1, 4, 5 and 6 . This could be due to a non-linear behaviour of the powder bed, with more intense flows between zones at the beginning of the blending operation and virtually no powder exchanges when the bed is consolidated after a few rotations of the blades. This behaviour cannot be predicted by a linear
Markov chain, in which the transition probabilities do not depend on transition number (and thus time).
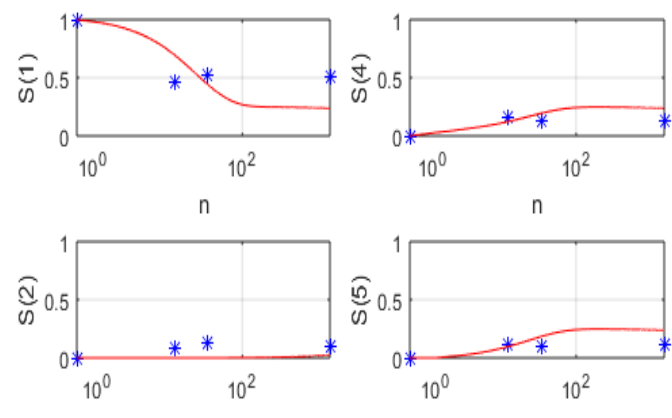

n
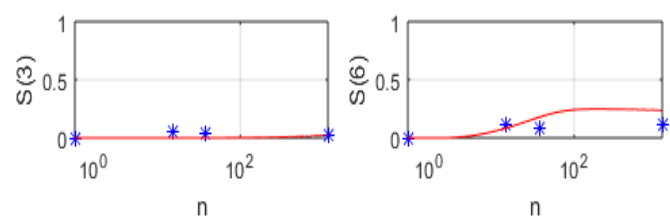

Fig. 7. Comparison of simulations and experimental data for lactose, with initial tracer position in zone 1

\section{Conclusion}

The influence of powder cohesion in convective blending has been characterized through mixing kinetics experiments. A simple model based on Markov chains is able to reproduce satisfactorily the self-mixing of a freeflowing powder. However, the non-linear behaviour of cohesive powders is more problematic and requires more advanced modelling.

The authors would like to thank Luana Grillet and Asma Toualoua for their valuable contribution to the experimental results.

\section{References}

1 L. Legoix, C. Gatumel, M. Milhe, H. Berthiaux, Rheology of cohesive powders in a pilot scale planetary blender, Powder Technol. 305 (2017).

2 K. Marikh, H. Berthiaux, V. Mizonov, E. Barantseva, D. Ponomarev, Flow Analysis and Markov Chain Modelling to Quantify the Agitation Effect in a Continuous Powder Mixer, Chem. Eng. Res. Des. 84 (2006).

3 C. Ammarcha, C. Gatumel, J.L. Dirion, M. Cabassud, V. Mizonov, H. Berthiaux, Transitory powder flow dynamics during emptying of a continuous mixer, Chem. Eng. Process. Process Intensif. 65 (2013).

4 M. Aoun-Habbache, M. Aoun, H. Berthiaux, V. Mizonov, An experimental method and a Markov chain model to describe axial and radial mixing in a hoop mixer, Powder Technol. 128 (2002). 